\title{
Spinal Granulocytic Sarcoma Manifesting as Radiculopathy in a Nonleukemic Patient
}

\author{
-Case Report- \\ Tomoo InOUE, Toshiyuki TAKAHASHI*, Hiroaki SHIMIZU*,
Masayuki KANAMORI, Toshihiro KUMABE,
Mika WATANABE**, and Teiji TOMINAGA \\ Departments of Neurosurgery and ${ }^{* *}$ Pathology, Tohoku University \\ Graduate School of Medicine, Sendai, Miyagi; \\ ${ }^{*}$ Department of Neurosurgery, Kohnan Hospital, Sendai, Miyagi
}

\begin{abstract}
A 26-year-old nonleukemic woman presented with lumbosacral granulocytic sarcoma manifesting as progressive low back pain and numbness of her left lower leg persisting for 3 months. Physical examination revealed hypesthesia within the left $\mathrm{S1}$ area of the sensory dermatome, decreased Achilles tendon reflex in the left lower extremity, and walking impairment due to severe pain in her left hip and leg. Magnetic resonance imaging confirmed an extradural mass in the spinal canal at the L5-S2 levels with invasion to the pelvis from the left sacral foramen. Positron emission tomography with $\left[{ }^{18} \mathrm{~F}\right]$ fluorodeoxyglucose (FDG-PET) showed hyperaccumulation indicating malignant tumor. Baseline laboratory data were normal. Decompressive laminectomy and tumor removal were performed. Histological examination identified granulocytic sarcoma. Bone marrow involvement was absent. She underwent adjuvant chemotherapy and radiotherapy, resulting in reduced residual lesion and neurological improvement. Immediate diagnosis and adequate systematic treatment are recommended for spinal granulocytic sarcoma in nonleukemic patients to prevent or delay progression to leukemia. The importance of immunohistochemical staining in the differential diagnosis from other types of spinal tumor, and the efficacy of FDG-PET for evaluation of the treatment are also emphasized.
\end{abstract}

Key words: granulocytic sarcoma, leukemia, immunohistochemistry, positron emission tomography, spine

\section{Introduction}

Granulocytic sarcoma originates from myeloid precursor cells and occurs in $0.7-9.0 \%$ of patients with acute or chronic myelogenous leukemia, myelodysplasia, and myeloproliferative disorders. ${ }^{1)}$ Granulocytic sarcoma was initially reported in $1811,{ }^{3)}$ and described as chloroma in 1853 due to the typical greenish-yellow appearance (from the Greek chloros, green). ${ }^{13)}$ Granulocytic sarcoma most commonly occurs in the soft tissues of the head and neck, bone, and skin, ${ }^{9}{ }^{9}$ and less often in the central nervous system and spinal cord. , $^{2,-7,9-12,14-28,30)}$

Granulocytic sarcoma seldom manifests before the identification of leukemia in the peripheral

Received July 12, 2007; Accepted November 28, 2007 blood or bone marrow. ${ }^{2,5-7,9-12,14-28,30)}$ The finding of granulocytic sarcoma in patients with leukemia may predict the first presentation of blast crisis. ${ }^{1)}$ On the other hand, granulocytic sarcoma may occur in the absence of leukemia. ${ }^{2,6,21)}$ The correct diagnosis of granulocytic sarcoma is obtained only in about $50 \%$ of nonleukemic patients due to its rareness and the histological and radiological similarities to malignant lymphoma or Ewing sarcoma. ${ }^{29)}$ Spinal granulocytic sarcoma in aleukemic patients is rare but important because late bone marrow involvement may be related to blast crisis and cause death. $6,14,16,18,20,24,26)$

We treated a nonleukemic patient with radiculopathy caused by spinal extradural granulocytic sarcoma. This case indicates the importance of immunohistochemistry in the differential diagnosis, and the efficacy of positron emission tomography 
with $\left[{ }^{18} \mathrm{~F}\right]$ fluorodeoxyglucose (FDG-PET) for evaluation of the treatment.

\section{Case Report}

A 26-year-old previously healthy woman presented with progressive low back pain and numbness of her left lower leg persisting for 3 months. She denied motor weakness and bowel or bladder incontinence.

Physical examination revealed hypesthesia within the left S1 area of the sensory dermatome, decreased Achilles tendon reflex in the left lower extremity, and walking impairment due to severe pain in her left hip and leg. Laboratory examinations were normal. Magnetic resonance (MR) imaging confirmed an extradural mass in the spinal canal at the L5-S2 levels with invasion to the pelvis from the left sacral foramen (Fig. 1A). FDG-PET indicated the lesion had a maximum standardized uptake value (SUV) of 7.8 (Fig. 2A). SUV indicates the tissue activity of FDG per unit body weight, calculated as (mean concentration of radioactivity in the region of interest)/(injected radioisotope activity/body weight) $\times$ calibration factor.

The patient underwent tumor removal for dural sac decompression through S1-2 laminectomy. A rubbery and grayish tumor was found in the epidural space, compressing the dural sac and left S1 root. The tumor in the intracanal space was totally removed. Histological examination demonstrated fibroadipose connective tissue including diffuse cellular infiltrates. Neoplastic cells with small round and hyperchromatic nuclei proliferated in a diffuse pattern (Fig. 3A). Intraoperative pathological assessment by frozen section suggested malignant lymphoma or Ewing sarcoma/primitive neuroectodermal tumor (PNET). Immunohistochemical staining showed the tumor cells were positive for myeloperoxidase (Fig. 3B), CD15 (Fig. 3C), neutrophil elastase (Fig. 3D), CD45 (Fig. 3E), and lysosome (Fig. $3 F$ ), but negative for CD3, CD79 $\alpha$, CD56, synaptophysin, neurofilament protein, CD1a, S-100 protein, cytokeratin, and CD99. The final histological diagnosis was granulocytic sarcoma.

Postoperative examination found no indications of bone marrow involvement on bone marrow aspiration. The patient underwent adjuvant chemotherapy for acute myeloid leukemia using cytarabine, idarubicin, and leuprorelin, followed by irradiation of the craniospinal deposits and bone marrow transplantation. Four months later, MR imaging showed disappearance of the intracanal tumor mass with marked reduction of the intraforaminal and extrasacral tumor (Fig. 1B), and FDG-PET demonstrated decreased maximum SUV of 2.4 (Fig. 2B). She

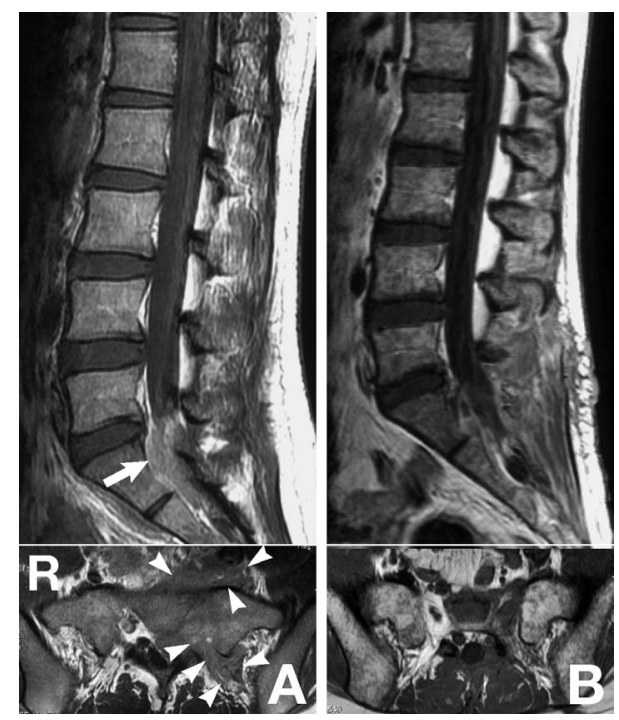

Fig. 1 A: $T_{1}$-weighted magnetic resonance (MR) images with gadolinium enhancement showing a slightly enhanced extradural mass (arrow) in the spinal canal at the L5-S2 levels with invasion to the extrasacrum through the left anterior and posterior sacral foramen (arrowheads). B: $T_{1}$-weighted MR images with gadolinium enhancement 4 months after the systemic chemotherapy and radiotherapy revealing disappearance of the intracanal tumor mass with marked reduction of the intraforaminal and extrasacral tumor.



Fig. 2 A: Positron emission tomography with $\left[{ }^{18}\right.$ F]fluorodeoxyglucose (FDG-PET) scan indicating the lesion had a maximum standardized uptake value (SUV) of 7.8 (arrow). B: FDG-PET scan 4 months after the systemic chemotherapy and radiotherapy showing decreased maximum SUV of 2.4 (arrow). 




Fig. 3 A: Photomicrograph showing neoplastic cells with small round and hyperchromatic nuclei proliferating in a diffuse pattern. Hematoxylin and eosin stain, original magnification $\times 40$. B-F: Photomicrographs demonstrating positive immunohistochemical staining for myeloperoxidase (B), CD15 (C), neutrophil elastase (D), CD45 (E), and lysosome (F). Original magnifications $x$ 40.

had achieved neurological improvement without graft versus host disease and MR imaging revealed no recurrence at 12 months after the transplantation.

\section{Discussion}

Twenty-six aleukemic patients with spinal granulocytic sarcomas, 23 men and three women aged 2 to 74 years (median, 33 years), have been reported to date (Table 1)..$^{2,5-7,9-12,14,16-28,30)}$ The male predominance could be associated with the higher incidence of acute myeloid leukemia in men. ${ }^{4)}$ The affected sites included two cases at cervical, 17 at thoracic, 10 at lumbar, and three at sacral spinal levels. The chief clinical findings were related to the affected sites and included pain in 17 patients, paresis or weakness in 21 , bowel or bladder incontinence in 13 , and sensory impairment such as hypesthesia or numbness in 16.

The treatment strategy will individually vary for nonleukemic patients with spinal granulocytic sarcoma. Surgical decompression was performed in 22 of 26 patients, and 21 of these received additional acute myeloid leukemia type therapies consisting of chemotherapy, radiotherapy, or bone marrow transplantation. The follow-up period varied, but the outcome was generally poor, especially in patients showing progression to leukemia. Eight patients developed acute leukemia, $, 614,16,18,20,25,26,30)$ and six patients died following leukemic relapse or graft versus host disease. ${ }^{6,14,16,18,20,26)}$ On the other hand, 13 of the 26 patients showed no bone involvement, ${ }^{2,5-7,9,11,12,17,27,28)}$ and only four patients died of chemotherapy or radiotherapy related clinical disorders. ${ }^{6,7,17,24)}$ This finding possibly reflects the aggressive initial treatment and prevention of progression to leukemia. Therefore, prompt diagnosis and adequate treatment are essential to achieve good outcome.

The histological diagnosis of granulocytic sarcoma is difficult in the absence of evidence of acute myeloid leukemia. The differential diagnosis includes malignant lymphoma, Ewing sarcoma/PNET, Langerhans histiocytosis $\mathrm{X}$, and metastatic tumors. ${ }^{1,3)}$ Granulocytic sarcoma consists of histologically uniform immature cells, but often includes mature neutrophils and immature eosinophils. ${ }^{12,19,22)}$ Therefore, immunohistochemistry is effective and should be performed to confirm the diagnosis of granulocytic sarcoma. Table 2 summarizes the differences in the immunohistochemical staining of tumors resembling granulocytic sarcomas. Myeloperoxidase, CD15 (granulocyte associate antigen), and neutrophil elastase are especially effective to differentiate granulocytic sarcoma from other tumors. CD99 (MIC2), synaptophysin, and neurofilament protein are positive for Ewing sarcoma/ PNET, but negative for granulocytic sarcoma. CD3, CD56, and $\operatorname{CD} 79 \alpha$ are useful for differentiating granulocytic sarcoma from malignant lymphoma. CD1a and S-100 protein are positive for Langerhans histiocytosis $\mathrm{X}$, and cytokeratin for metastatic tumor, but all are negative for granulocytic sarcoma.

In the present case, we used FDG-PET to estimate the malignancy of the tumor and the efficacy of the treatment. MR imaging with gadolinium enhancement is considered to consistently slight enhance the tumor mass, but cannot evaluate the metabolism. On the other hand, PET can estimate the metabolism of tumors in vivo using various positron tracers. ${ }^{8)}$ Increased uptake and consumption of deoxyglucose is related to aerobic glycolysis in tumors. FDG-PET is considered to be sensitive to detect this process because malignant tumors have increased glucose metabolism. ${ }^{8)}$ In our case, FDG-PET successfully identified the metabolically active lesion and suggested the high malignancy, and demonstrated decreased uptake after chemotherapy and radiotherapy. This follow-up FDG-PET may indicate that there was reduction in tumor cellularity due to the treatment. However, it is difficult to regard the findings as complete remission because, to the best of our knowledge, any previous reports about spinal granulocytic sarcoma did not evaluate the lesions by FDG-PET.

The malignancy cannot be investigated by 


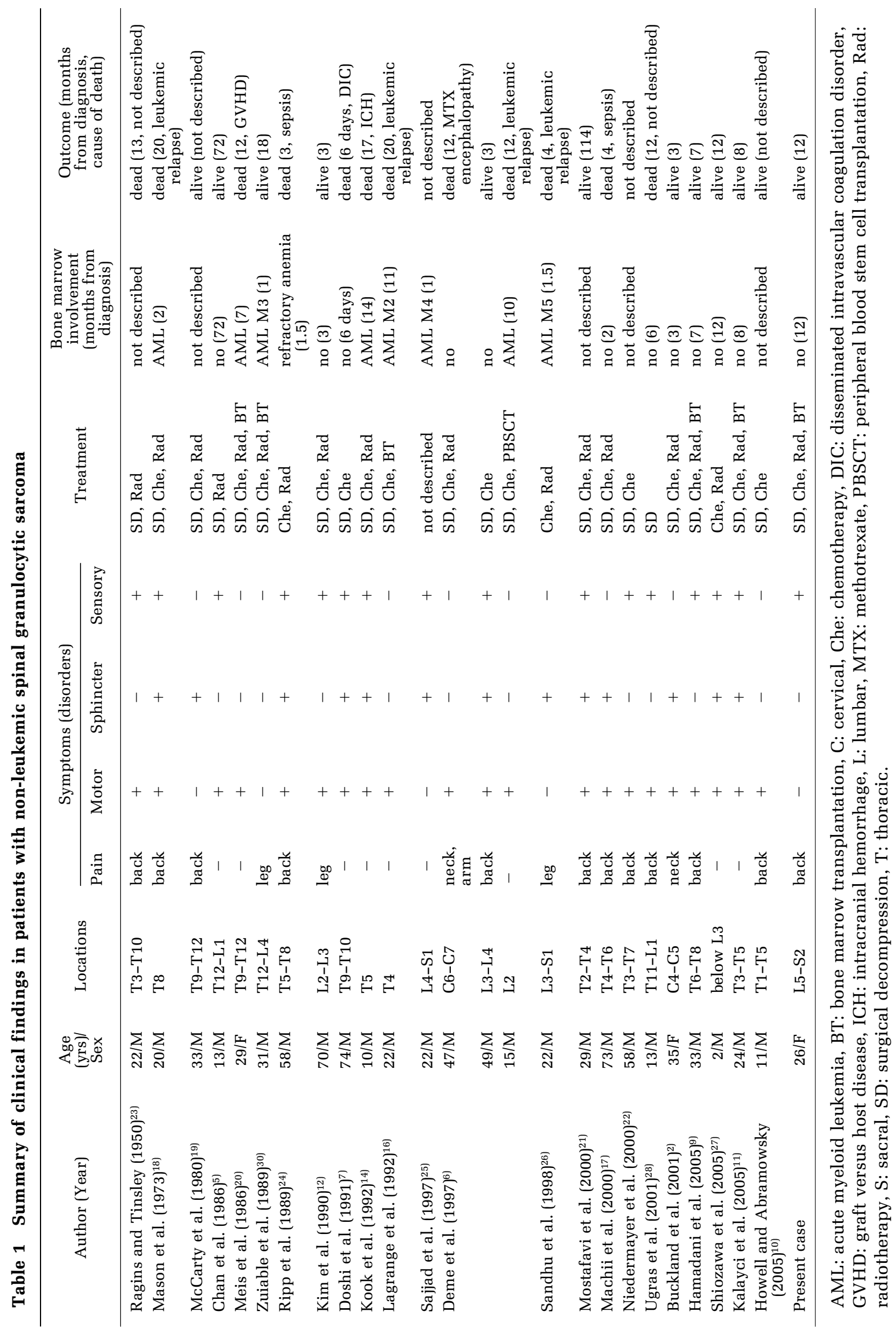


Table 2 Characteristics of immunohistochemical staining in tumors resembling granulocytic sarcoma

\begin{tabular}{lccccc}
\hline & $\begin{array}{c}\text { Granulocytic } \\
\text { sarcoma }\end{array}$ & $\begin{array}{c}\text { Malignant } \\
\text { lymphoma }\end{array}$ & $\begin{array}{c}\text { Ewing sarcoma/ } \\
\text { PNET }\end{array}$ & $\begin{array}{c}\text { Langerhans } \\
\text { histiocytosis X }\end{array}$ & $\begin{array}{c}\text { Carcinoma } \\
\text { metastasis }\end{array}$ \\
\hline Myeloperoxidase & + & - & - & - & - \\
CD15 & + & - & - & - & - \\
Neutrophil elastase & + & - & - & - & - \\
CD45 & + & + & - & + & - \\
Lysosome & + & - & - & - & - \\
CD3 & - & + & - & - & - \\
CD79 $\alpha$ & - & + & - & - & \pm \\
CD56 & - & - & + & - & \pm \\
Synaptophysin & - & - & - & + & - \\
Neurofilament protein & - & - & - & - & - \\
CD1a & - & - & + & - & + \\
S-100 protein & - & - & + & & - \\
Cytokeratin & - & - & & - & - \\
CD99 & - & & - & & - \\
\hline
\end{tabular}

PNET: primitive neuroectdermal tumor.

peripheral blood examination or cerebrospinal fluid cytology in aleukemic patients, but PET can provide useful independent and complementary metabolic information. The present case indicates that the combination of MR imaging and FDG-PET allows detection of primary spinal malignant tumor and comprehensive evaluation of treatment response.

Granulocytic sarcoma is rare, and clinical diagnosis is especially difficult without bone marrow involvement. Spinal granulocytic sarcoma should be included in the differential diagnosis of spinal lesion. Adequate systemic therapy should be initiated to prevent blast crisis. Immunohistochemistry can establish the final diagnosis of granulocytic sarcoma.

\section{References}

1) Brunning RD: Ackerman's Surgical Pathology. St Louis, Mosby-Year Book, 1996, pp 1821-1828

2) Buckland ME, Scolyer RA, Donellan MB, Brew $S$, McGee-Collett M, Harper CG: Spinal chloroma presenting with triplegia in an aleukaemic patient. Pathology 33: 386-389, 2001

3) Burns A: Observations of Surgical Anatomy, Head and Neck. Edinburgh, Thomas Royce and Co, 1811, pp 364-366

4) Cartwright RA, Gurney KA, Moorman AV: Sex ratios and the risks of haematological malignancies. $\mathrm{Br} \mathrm{J}$ Haematol 118: 1071-1077, 2002

5) Chan JK, Lau WH, Saw D: Extradural granulocytic sarcoma of the spine: a unique case of long survival after local therapy. Am J Hematol 22: 439-441, 1986

6) Deme S, Deodhare SS, Tucker WS, Bilbao JM: Granulocytic sarcoma of the spine in nonleukemic patients: report of three cases. Neurosurgery 40:
1283-1287, 1997

7) Doshi HM, Schochet SS Jr, Gold M, Nugent GR: Granulocytic sarcoma presenting as an epidural mass with acute paraparesis in an aleukemic patient. Am J Clin Pathol 95: 228-232, 1991

8) Go KG, Pruim J, Que TH, Vaalburg W, HaaxmaReiche H: Evaluation of dissemination studies with FDG whole-body positron emission tomography in patients with suspected metastatic tumours of brain and spine. Acta Neurochir (Wien) 142: 627-631, 2000

9) Hamadani M, Tfayli A, Sethi S, Awab A, Hamdani N: Granulocytic sarcoma manifesting as multiple skeletal lesions. Am J Med Sci 330: 139-143, 2005

10) Howell DL, Abramowsky CR: Granulocytic sarcoma presenting as a large mediastinal mass with acute spinal cord compression in a non-leukemic child. Pediatr Blood Cancer 44: 527, 2005 (letter)

11) Kalayci $M$, Sumer $M$, Yenidunya $S$, Ozdolap $S$, Acikgoz B: Spinal granulocytic sarcoma (chloroma) presenting as acute cord compression in a nonleukemic patient. Neurol India 53: 221-223, 2005

12) Kim FS, Rutka JT, Bernstein M, Resch L, Warner E, Pantalony D: Intradural granulocytic sarcoma presenting as a lumbar radiculopathy. Case report. J Neurosurg 72: 663-667, 1990

13) King A: A case of chloroma. Monthly J Med 17: 97, 1853

14) Kook H, Hwang TJ, Choe K, Yang DW, Nam JH, Park CS: Spinal epidural granulocytic sarcoma preceding acute myelogenous leukemia. J Korean Med Sci 7: 291-296, 1992

15) Krause JR: Granulocytic sarcoma preceding acute leukemia: a report of six cases. Cancer 44: 1017-1021, 1979

16) Lagrange $M$, Gaspard MH, Lagrange JL, Michiels JF, Hofman P, Thyss A, Schneider M: Granulocytic sarcoma with meningeal leukemia but no bone marrow involvement at presentation. A report of two cases 
with characteristic cerebrospinal fluid cytology. Acta Cytol 36: 319-324, 1992

17) Machii R, Muto A, Okano Y, Akizuki M, Katsumata Y: [Granulocytic sarcoma presenting as an epidural mass with spinal cord compression]. Rinsho Ketsueki 41: 653-657, 2000 (Jpn, with Eng abstract)

18) Mason TE, Demaree RS Jr, Margolis CI: Granulocytic sarcoma (chloroma), two years preceding myelogenous leukemia. Cancer 31: 423-432, 1973

19) McCarty KS Jr, Wortman J, Daly J, Rundles RW, Hanker JS: Chloroma (granulocytic sarcoma) without evidence of leukemia: facilitated light microscopic diagnosis. Blood 56: 104-108, 1980

20) Meis JM, Butler JJ, Osborne BM, Manning JT: Granulocytic sarcoma in nonleukemic patients. Cancer 58: 2697-2709, 1986

21) Mostafavi H, Lennarson PJ, Traynelis VC: Granulocytic sarcoma of the spine. Neurosurgery 46: 78-84, 2000

22) Niedermayer I, Schmitt-Graeff A, Kolbel CB, Hertel F, Feiden W: [Granulocytic sarcoma (so-called chloroma) as a possible cause of spinal cord compression. Case report and differential diagnosis]. Pathologe 21: 82-85, 2000 (Ger, with Eng abstract)

23) Ragins AB, Tinsley M: Chloroma; report of a case. J Neuropathol Exp Neurol 9: 186-192, 1950

24) Ripp DJ, Davis JW, Rengachary SS, Lotuaco LG, Watanabe IS: Granulocytic sarcoma presenting as an epidural mass with cord compression. Neurosurgery 24: 125-128, 1989

25) Sajjad Z, Haq N, Kandula V: Case report: granulocyt- ic sarcoma (GS) presenting as acute cord compression in a previously undiagnosed patient. Clin Radiol 52: 69-71, 1997

26) Sandhu GS, Ghufoor K, Gonzalez-Garcia J, ElexpuruCamiruaga JA: Granulocytic sarcoma presenting as cauda equina syndrome. Clin Neurol Neurosurg 100: 205-208, 1998

27) Shiozawa Y, Kiyokawa N, Saito M, Fujimoto J, Hata J, Yamashiro Y: Granulocytic sarcoma of the spine in a child without bone marrow involvement: a case report and literature review. Eur J Pediatr 164: 616-620, 2005

28) Ugras S, Cirak B, Karakok M, Guven B: Spinal epidural granulocytic sarcoma (chloroma) in a nonleukemic child. Pediatr Int 43: 505-507, 2001

29) Williams MP, Olliff JF, Rowley MR: CT and MR findings in parameningeal leukaemic masses. J Comput Assist Tomogr 14: 736-742, 1990

30) Zuiable A, Aboud H, Nandi A, Powles R, Treleaven J: Extramedullary disease initially without bone marrow involvement in acute promyelocytic leukaemia. Clin Lab Haematol 11: 288-289, 1989

Address reprint requests to: Tomoo Inoue, M.D., Department of Neurosurgery, Tohoku University Graduate School of Medicine, 1-1 Seiryo-machi, Aoba-ku, Sendai 980-8574, Japan.

e-mail: tomoo49@nsg.med.tohoku.ac.jp 